\title{
THE THORACIC LIMB OF THE SURICATE (SURICATA SURICATTA): OSTEOLOGY, RADIOLOGIC ANATOMY, AND FUNCTIONAL MORPHOLOGIC CHANGES
}

\author{
Sheryl L. van Staden, B.V.Sc., M.Med.Vet. (Rad.), Dipl. E.C.V.D.I.
}

\begin{abstract}
The purpose of the study was to identify unique features of the normal osteology and radiologic anatomy of the thoracic limb of the meerkat or suricate (Suricata suricatta), as no comprehensive information has been published. Bone specimens of 19 suricates were studied. Individual bones of the manus of one preserved carcass were studied in situ. Radiographic evaluation was performed in six animals. Comparisons to domestic carnivores were made and functional morphologic changes were identified. A suprahamate process was present on the scapula spine. Both supratrochlear and supracondylar foramina were present in the distal humerus, with a small Fossa coronoidea seen cranially. The medial epicondyle was markedly larger than the lateral epicondyle. The Tuberositas radii was located caudally. The proximal end of the olecranon was prominent medially. The large medial coronoid process had an extensive proximal articulation facet for the humeral trochlea. The ulna styloid process articulated with the ulnar and accessory carpal bones. The manus was similar to that of domestic carnivores; however, Os metacarpalis I was markedly reduced with the absence of the first digit. There were seven carpal bones. Os carpi radiale was the largest, with a large palmaro-medial process and a small sesamoid bone present medially. Ossa metacarpalia II-V and corresponding phalanges were slender, with an elongated Processus unguicularis (third phalanx) present. Radiologic findings demonstrated increased mobility of the scapula and shoulder joint, with ease of abduction. On the cranio-caudal view of the humerus and elbow joint, the distal radius and manus were consistently rotated in a supinated position. Morphologic changes were identified for the enhanced, predominant function of the shoulder, elbow, carpal, and digital flexor muscles associated with superior digging ability, and supination of the antebrachium and manus. Reference values for size parameters of the long bones are reported. The findings will assist in clinical zoologic medicine and paleozoologic studies.
\end{abstract}

Key words: Anatomy, osteology, radiology, Suricata suricatta, suricate, thoracic limb.

\section{INTRODUCTION}

Current zoologic descriptions are limited to basic anatomic characteristics of the meerkat or suricate (Suricata suricatta). ${ }^{4,6}$ The species is described as digitigrade but well designed for standing erect, with distinguishing features such as the enlarged curved claws and the absence of the first digit on the manus. Although the natural habitat of suricates is limited to southern Africa, suricates are widespread internationally in zoologic gardens. Imaging studies of the musculoskeletal system are now relatively commonly performed in clinical zoologic medicine. ${ }^{5}$ No comprehensive information has, however, been published on the normal osteology and radiologic anatomy of the suricate. This study provides a morphologic description of selected unique features of the normal osteology and correlative radiologic anatomy of the thoracic limb of the suricate, in comparison to domestic carnivores.

From the Department of Anatomy and Physiology, Faculty of Veterinary Science, University of Pretoria, Private Bag X04, Onderstepoort, 0110, Republic of South Africa (van Staden). Correspondence should be directed to Dr. van Staden (vip@pop.co.za).
Concurrent observation of function has identified specific functional morphologic changes.

\section{MATERIALS AND METHODS}

A prospective morphologic study of the thoracic limb of $S$. suricatta was carried out on bone specimens and radiographs, using terms as per Nomina Anatomica Veterinaria. ${ }^{3}$

Bone specimens in museum zoologic collections, derived from 19 individual animals (17 mature), were examined to establish the normal osteology. In addition, the manus of both thoracic limbs of a preserved adult carcass were dissected and the bones studied in situ. Each individual bone and the related topography were described. A total of 32 scapulae, 35 humeri, 29 radii and ulnae, and the skeletal components of the manus region (two complete specimens and selected bones from 19 animals) were evaluated.

Five live captive male animals (age range 11-36 mo old) under general anesthesia and one preserved carcass (mature male) were used for the radiographic study. Anesthesia was achieved by intramuscular injection of midazolam (Dormicum $^{\circledR}$, Roche, Basel, 4070, Switzerland) and tiletamine/zolazepam combination (Zoletil ${ }^{\circledR}$, Virbac, 
B.P. 27, Carros Cedex, 06511, France) followed by inhalation anesthesia (isoflurane, Piramal, Mumbai, 400 013, India) as required. Standard anesthetic monitoring and radiographic safety precautions were applied. Digital radiographs were made with a high-output rotating anode Xray machine (SA Philips [Pty] Ltd., Johannesburg, Gauteng, 2000, South Africa) using a tabletop technique and cassettes with a mammography imaging plate (Fujifilm IP, Fujifilm Corporation, Johannesburg, Gauteng, 2000, South Africa). Processing was done on an indirect digital imaging system (Fujifilm FCR, Fujifilm Corporation). Medio-lateral (ML) and cranio-caudal ( $\mathrm{CrCd})$, caudo-cranial $(\mathrm{CdCr})$, or dorso-palmar (DPa) radiographic views were made of one entire thoracic limb in each of the animals. Additional images were made of the severed thoracic limbs and digits from the carcass. A detailed study of the normal correlative radiologic anatomy was carried out. The impact of positioning during radiography on the radiologic appearance of various structures was determined.

Size parameters of long bones were measured in millimeters on both bone specimens and radiographs to establish reference values. Bones were measured using calipers. Radiographic measurements were done using the standard measurement tool of the digital system. Length was measured from proximal to distal extremities of the bone and on ML views. Width and breadth were measured at the midbody region of the bone and on $\mathrm{CrCd} / \mathrm{DPa}$ views and ML views, respectively. Statistical assessment of corresponding measurements (bone and radiographs) using a two-tailed paired $t$-test was performed (MS Excel ${ }^{\circledR}$, Microsoft Office, Microsoft, Johannesburg, Gauteng, 2000 , South Africa). Where no significant differences $(P>0.05)$ were found, all measurements were included in one group and the mean, range, and standard deviation for each value were calculated.

Osteologic and radiologic findings were also compared to those of the domestic cat (Felis catus) and $\operatorname{dog}$ (Canis familiaris), and other currently available information on related wild carnivores. Normal function of the suricate's thoracic limbs was described by personal observation of the video recording of the suricate's natural behavior. ${ }^{2}$ Specific morphologic changes of the bones were identified and correlated to the specialized function observed.

This study was approved by the Animal Use and Care Committee of the Faculty of Veterinary
Science, University of Pretoria, and the National Zoological Gardens.

\section{RESULTS AND DISCUSSION}

\section{Scapula and proximal humerus}

Osteology: The scapula was elongated and narrower than that of the cat. The Angulus cranialis was poorly defined but the Angulus caudalis was acute with a beak-like caudal projection. The Margo caudalis was straight and thickened, forming a prominent ridge raised above the level of the adjacent deep Fossa infraspinata. Proximally, the caudal border had a flattened site for origin of $M$. teres major. The acromion of the Spina scapulae had a caudally directed, large Processus suprahamatus, which was square, elongated, or spatula-like. This process in the cat, however, has a more pointed tip. The Proc. hamatus was narrow, pointed, and cylindrical. There was a deep Incisura scapula with a well-defined Collum scapulae. The Tuberculum supraglenoidale was prominent and projected cranio-medially. The Proc. coracoideus was reduced to a small rounded projection. In contrast, this process is sharp and well defined in the cat. The Tub. infraglenoidale was reduced to a small roughened area caudally on the neck. The Clavicula was absent in the suricate, in contrast to the well-developed clavicle in the domestic cat (Fig. 1).

The morphology of the major and minor tubercles of the humerus in the suricate was similar to that in the cat. The Facies m. infraspinati was a well-defined, deep oval depression on the caudo-lateral aspect of the major tubercle. This depression is wide and shallow in the cat. The minor tubercle was a prominent, oval to rectangular protuberance medially. The Tuberositas teres minor was a well-defined convex protuberance, and relatively more prominent than in the cat. The Tuberositas teres major was an elongated broad ridge on the medial surface of the proximal humerus. The Tuberositas deltoidea was prominent and elongated (Fig. 2).

Shoulder positioning and radiologic anatomy: Positioning of the thoracic limbs demonstrated the great mobility of the scapula and shoulder joint. With the limb fully extended cranially, all the borders of the scapula, the caudal angle, the hamate process of the acromion, and the major tubercle (humerus) were all clearly seen on the ML view. The extension of the thoracic limb cranially while in dorsal recumbency (Fig. 3) resulted in an oblique $\mathrm{CdCr}$ view of the scapula 


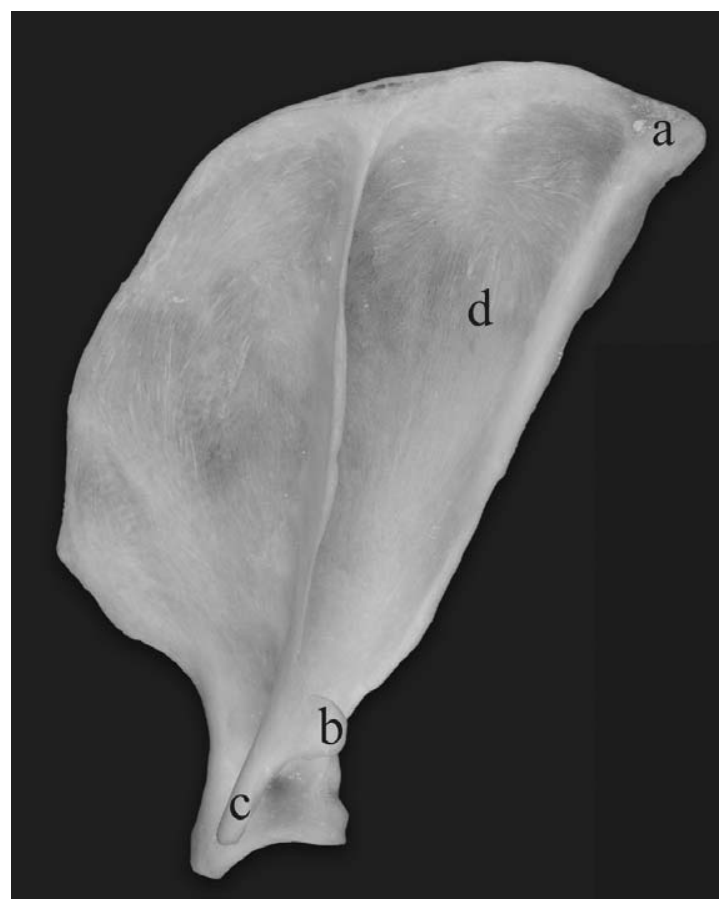

Figure 1. Lateral surface of the scapula showing thickened caudal border. a. Beak-like caudal angle. b. Suprahamate process. c. Hamate process. d. Fossa infraspinatus.

with the caudal border facing laterally in the image. The origin of the suprahamate process was seen distally on the scapular spine, ending in a clearly defined tip. This process was not visible on the ML view. Disto-medial to the humeral head, the minor tubercle with the proximal beak-like tip was well defined (Fig. 4). An additional view of the scapula was achieved with the shoulder maximally flexed (Fig. 3). In this position, there was marked abduction of the shoulder and humerus from the thoracic wall, and the mobile scapula was tilted obliquely to the radiographic cassette, resulting in some radiographic distortion. Despite superimposition of the distal scapula and proximal humerus, the hamate process could be seen extending beyond the humeral head. The narrow rounded summit of the major tubercle was clearly seen lateral to the humeral head, with a deep concavity laterally representing the Facies $\mathrm{m}$. infraspinati. The deltoid tuberosity was prominent laterally (Fig. 5). No mineralized remnant of the clavicle was seen radiographically in this study, in contrast to the small remnant, which is routinely detected radiographically in some dogs, medial to the shoulder joint.

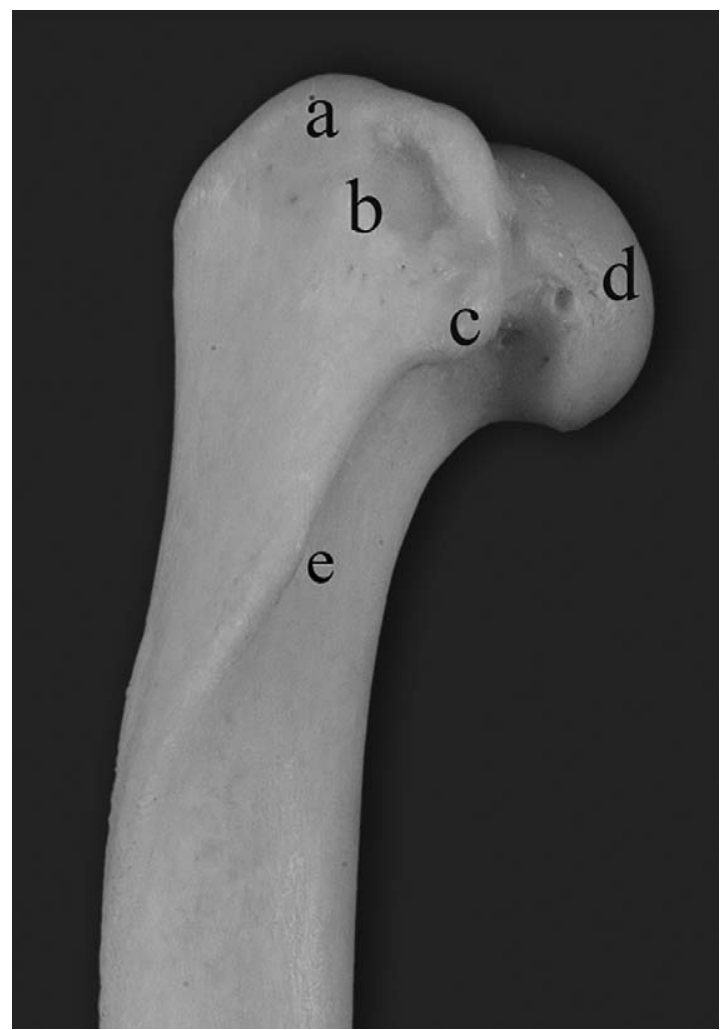

Figure 2. Lateral aspect of proximal humerus. a. Tub. majus. b. Facies $m$. infraspinati (to right). c. Tuberositas teres minor. d. Humeral head. e. Tuberositas deltoidea.

\section{Distal humerus and elbow}

Osteology: The distal extremity, Condylus humeri, consisted of both the Trochlea humeri and the Capitulum humeri (Fig. 6). The trochlea, for articulation with the ulna, had a prominent medial rim. The capitulum, for articulation with the radial head, was a wide cranio-lateral extension of the articulation surface, but was poorly demarcated from the trochlea and not as well defined as in the cat. The Epicondylus medialis was large, convex, irregular, and cranio-caudally flattened. The distal border was irregular, because of the origin sites for the carpal and digital flexor muscles and ligament attachment. It was approximately three times larger than the relatively small Epicondylus lateralis. There is a smaller size difference in the dog and the cat. The lateral aspect of the Fossa olecrani (60-70\% of the fossa size) was perforated by a large, oval Foramen supratrochleare (as in the dog). This Foramen joined the olecranon fossa to the shallow cranially located Fossa radialis. The Fossa coronoidea was 


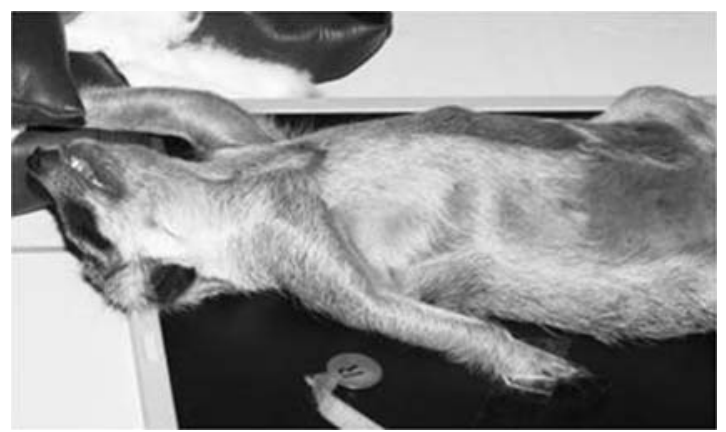

Figure 3. Radiographic positioning of the thoracic limbs, with maximal right shoulder flexion for the cranio-caudal view of the humerus.

also present cranially as a poorly defined small depression, medial to the supratrochlear foramen. On flexion of the elbow, the medial coronoid process of the ulna was accommodated by the coronoid fossa. This fossa was much smaller than the radial fossa in the suricate but is of a similar size in the cat. There was also an oval elongated For. supracondylare (similar to the cat) (Fig. 6). The presence of both the supratrochlear and supracondylar foramina in the suricate is similar to that reported for the Indian mongoose (Herpestes auropunctatus). ${ }^{1}$

Radiologic anatomy: The $\mathrm{CrCd}$ view of the elbow region (Fig. 5) demonstrated the large, triangular-shaped medial epicondyle, as it expanded from the distal humerus. The well-defined supratrochlear foramen was visualized adjacent to the superimposed proximal ulna. The humeral trochlea was seen medially with a shallow groove and a smooth transition to the extended flattened zone of the capitulum on the lateral aspect. The supracondylar foramen could not be seen on the true $\mathrm{CrCd}$ view. However, when the thoracic limb was extended cranially for the $\mathrm{CdCr}$ view of the shoulder, the brachium was pronated and the resultant oblique view of the elbow region allowed visualization of the supracondylar foramen (Fig. 4).

\section{The antebrachium}

Supination of the antebrachium and manus: To limit the anesthetic time and radiation exposure of the live animals, a single radiographic position and exposure was selected to provide a CrCd view of the brachium and antebrachium and a DPa view of the manus. There was a natural supination of the antebrachium and manus when the animal was placed as such in dorsal recumbency with a flexed shoulder. As a result, the humerus and

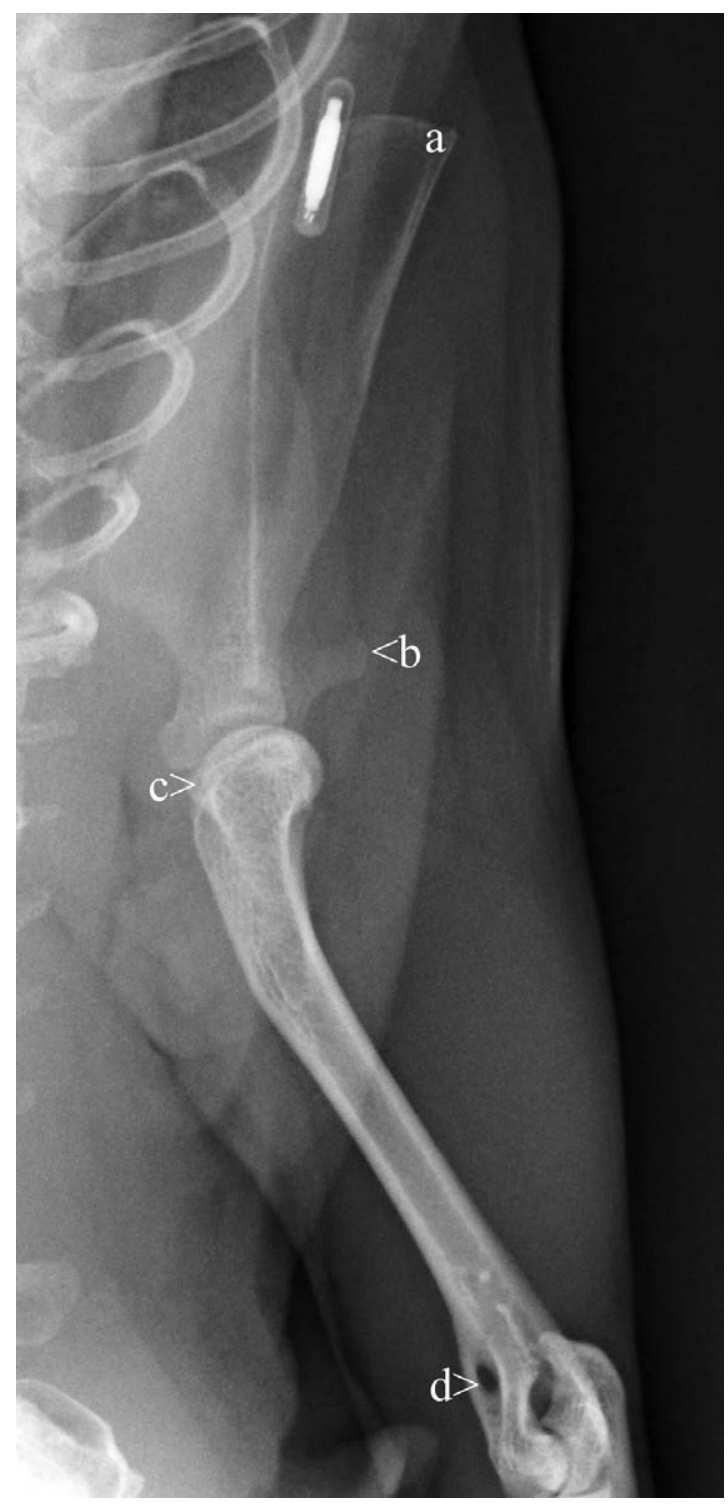

Figure 4. Caudo-cranial view of the shoulder. a. Caudal angle of the scapula. b. Proc. suprahamatus seen laterally. c. Tub. minus. d. Supracondylar foramen.

elbow joint were imaged in a true $\mathrm{CrCd}$ position but the manus (and distal radius-ulna) was consistently demonstrated in a relatively supinated position in all the radiographs, creating an oblique DPa (supinated) view (Fig. 7).

Most of the structural components of the radius and ulna of the suricate and the relative size of these two bones were similar to those of the cat. On the CrCd view, the midbody of the radius had a clear lateral bowing. The ulna lay more medially at the elbow joint. In the mid and distal antebra- 


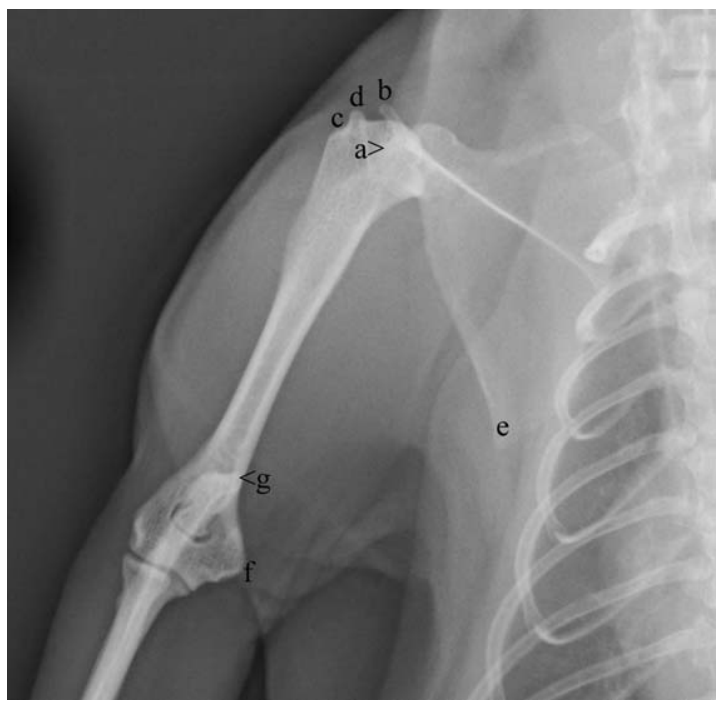

Figure 5. Cranio-caudal view of the humerus and elbow, showing mobility of the scapula and abduction of the shoulder. a. Proc. suprahamatus. b. Proc. hamatus. c. Deep Facies m. infraspinati. d. Tub. majus. e. Caudal angle of scapula. f. Large medial epicondyle. g. Medial tubercle of olecranon.

chium, the radius and ulna were superimposed on each other, but the styloid process of the ulna emerged again laterally to the distal radius towards the carpal region.

The ulna: The cranial part of the olecranon was widest, and had a central depression bordered by two ridges dorsal to the Proc. anconeus. The medial ridge was most prominent and formed an elongated prominent tubercle, which extended further proximal than the Tuber olecrani. This medial tubercle was well demonstrated on the $\mathrm{CrCd}$ view of the elbow, where it was superimposed over the distal humerus in the region of the supracondylar foramen (Fig. 5). In the cat, the olecranon has a medial and lateral tubercle of similar size cranially. In the dog, there are two prominent tubercles dorsal to the anconeal process, but the Tuber olecrani forms the highest point caudally. In the suricate, the Tuber olecrani was relatively less prominent than is seen in the cat. The medial surface of the olecranon was deeply concave in the suricate. The anconeal process was beak shaped, and blunted when compared to the dog. The Proc. coronoideus medialis was wedge shaped and markedly larger than the lateral coronoid process. The sharp tip projected beyond the cranial margin of the ulna. In the domestic cat, the tip is hook-like. On the $\mathrm{CrCd}$ view of the elbow, the medial coronoid

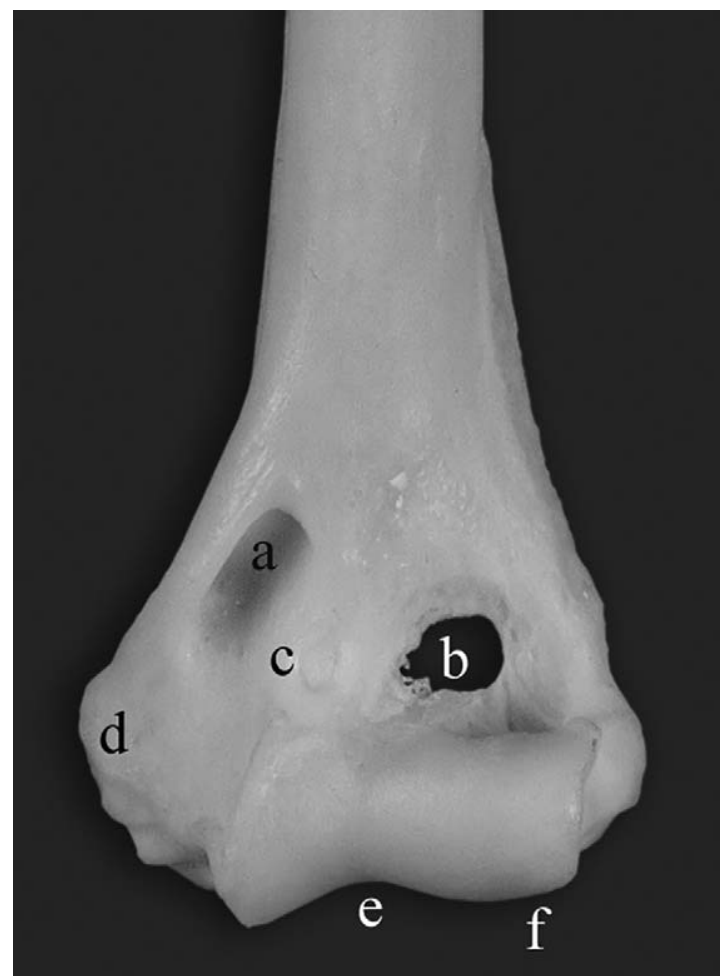

Figure 6. Cranial aspect of the distal humerus. a. Supracondylar foramen. b. Supratrochlear foramen. c. Small fossa coronoidea (to right). d. Medial epicondyle. e. Trochlea. f. Capitulum.

process formed a well-defined triangular tip at the proximo-medial aspect of the ulna, where it articulated with the humeral trochlea. The small lateral coronoid process was flattened craniocaudally and projected laterally, ending in a thinblunted tip. The proximal facet of the medial coronoid process for articulation with the humeral trochlea was much larger than that of the lateral coronoid process in the suricate, as seen in the cat. Distal to the medial coronoid process, there was a well-defined oval roughened zone on the proximal ulna (the so-called "ulnar tuberosity"), for insertion of M. brachialis. The styloid process was the main component of the distal ulna and articulated with the ulna and accessory carpal bones. On the ML view, the supination of the antebrachium displaced the distal ulna (and styloid process) more caudally in relation to the radial trochlea.

The radius: The radius lay in a supinated position such that the Facies cranialis faced cranio-laterally and the Margo interosseus was located on the caudolateral aspect of bone. The well-defined Tuberositas radii was a flattened, elongated oval tuberosity projecting caudally 


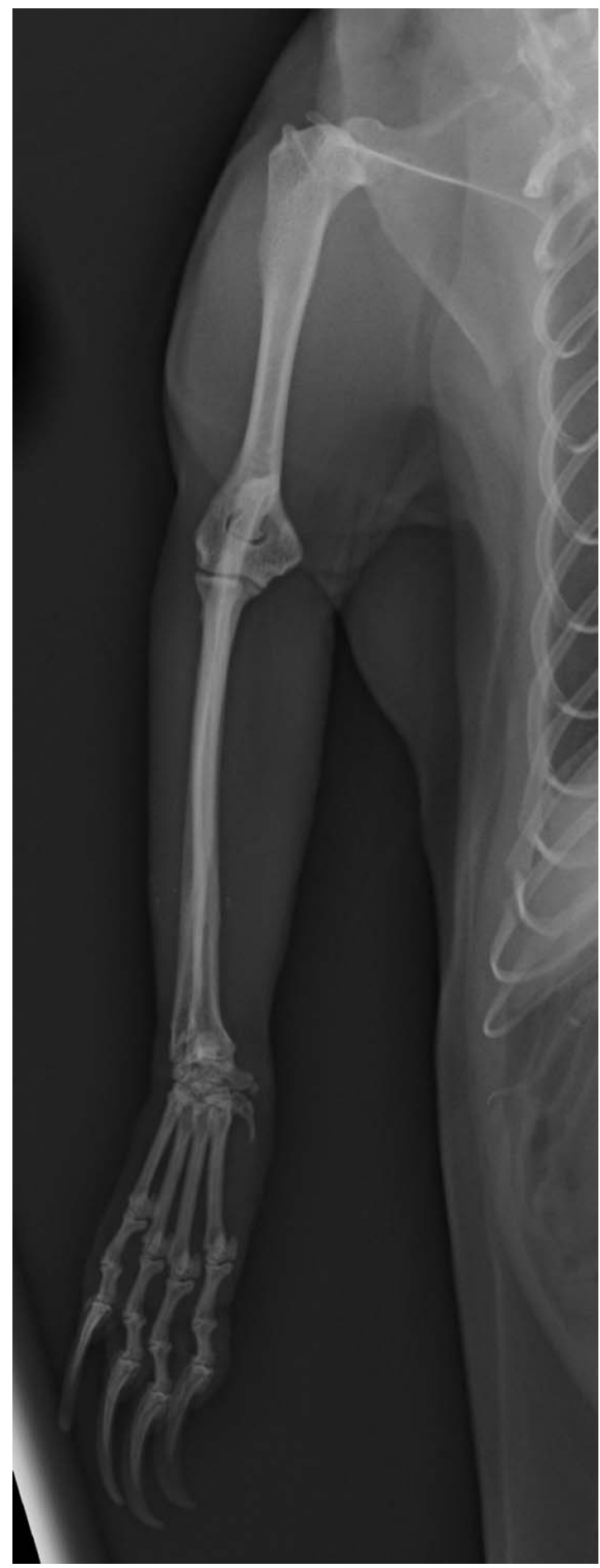

Figure 7. Cranio-caudal view of the humerus with concurrent supination of the antebrachium, resulting in a supinated oblique view of the manus. distal to the neck of the radius. This tuberosity was relatively more prominent and well defined in the suricate than in the domestic dog and cat. The distal extremity of the radius, the Trochlea radii, had a medially located wedge-shaped Proc. styloideus directed distally. The distal articulation facets for the radial and ulnar carpal bones could be distinguished. There were four distinct longitudinal grooves divided by crests on the cranial aspect of the trochlea, including a deep, sagittally directed groove (for the tendon of $\mathbf{M}$. extensor carpi radialis), with a prominent crest laterally.

\section{The manus}

Additional true DPa view radiographs were made of the manus using isolated thoracic limbs, for clear assessment and description of the individual bones and associated joint spaces (including distal radius and ulna). A true DPa view of the distal thoracic limb of a suricate can, however, be achieved, by placing the animal in sternal recumbency, with the limb extended cranially, along with slight pronation of the elbow region.

The carpus: As in the dog and cat, there were seven carpal bones in the suricate (Figs. 8, 9). The radial, ulna, and accessory carpal bones were located in the proximal row. The distal row consisted of the ossa carpale I-IV. Os sesamoideum $\mathrm{m}$. abductoris digiti I longus was seen radiographically at the disto-medial aspect of the radial carpal bone and measured $1 \mathrm{~mm}$ in diameter. The articulation pattern of the various carpal bones was well demonstrated on the true DPa view of the carpus (Figs. 8, 10), except for the Os carpi accessorium, which was superimposed on the ulna carpal bone, and Os carpale I, which was seen more clearly on the supinated view of the manus (Fig. 11). Os carpi radiale was the largest of the carpal bones with an elongated process at the palmaro-medial aspect. One-third of this bone extended medially beyond the styloid process of the distal radius. In the domestic dog, this medial extension is much less pronounced and, in the domestic cat, the medial aspect of the radial carpal bone is at a similar level to the radial styloid process. There was a large, convex facet proximally for articulation with distal radius, and proximo-laterally a small facet for the ulna carpal bone. The distal concave surface presented various facets for articulation with each of the carpal bones in the distal row. Os carpi ulnare was smaller and lay obliquely at the lateral side. Os carpi accessorium had a flattened palmar extremity. The base articulated with the styloid process 


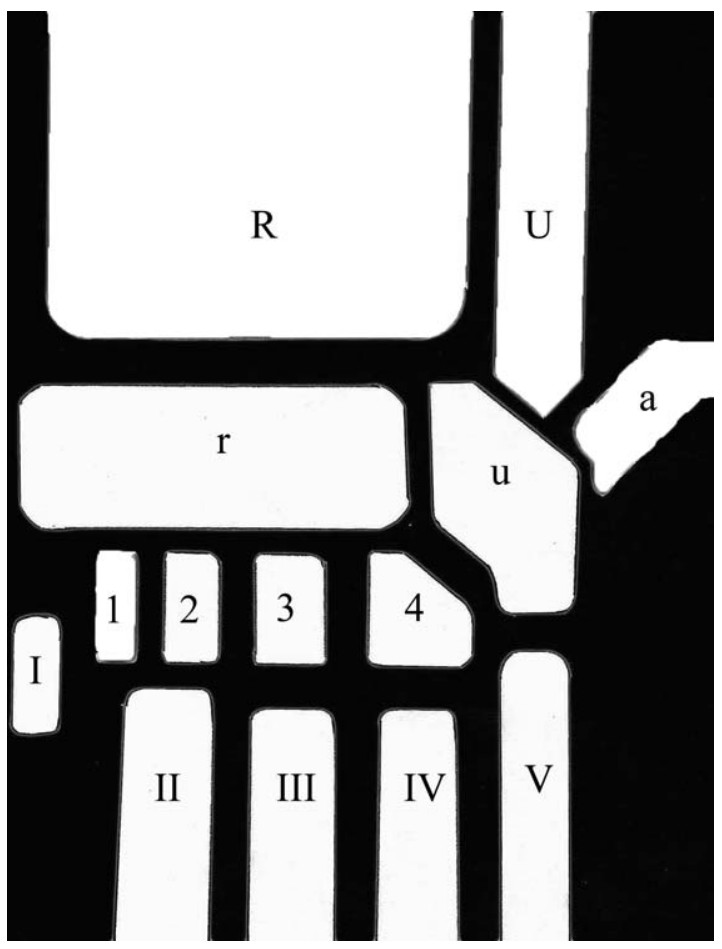

Figure 8. Schematic representation of the articulation pattern of the carpal bones. $\mathrm{R}$ indicates radius; $\mathrm{U}$, ulna; r, radial carpal bone; u, ulnar carpal bone; a, accessory carpal bone, 1-4, carpal bones 1-4; and I-V, Ossa metacarpalia I-V.

of the ulna (dorso-proximally) and the ulnar carpal bone. The fourth carpal bone was a large, wedge-shaped bone (as seen from the dorsal aspect), with a distal base. Os carpale III had an hourglass appearance as was seen radiographically (DPa view) and articulated with Os metacarpalis (Mc) III (distally). In comparison, the third carpal bone articulates with third and fourth metacarpal bones in the cat. Os carpale II was proximo-distally compressed and flattened. The first carpal bone was the smallest.

The metacarpus: Mc I was reduced to a small remnant oval-shaped bone, a few millimeters in length. In addition, there was a complete absence of the first digit. Mc II-V had similar features and structure. The body of each metacarpal bone was elongated and slender. The proximal bases articulated with the distal row of carpal bones and the adjacent metacarpal bones. The base of Mc II and the associated carpo-metacarpal joint space was situated most proximally in relation to the rest of the metacarpal bones. The base of $\mathrm{Mc} \mathrm{V}$ was narrowest dorsally but widened palmarly with a

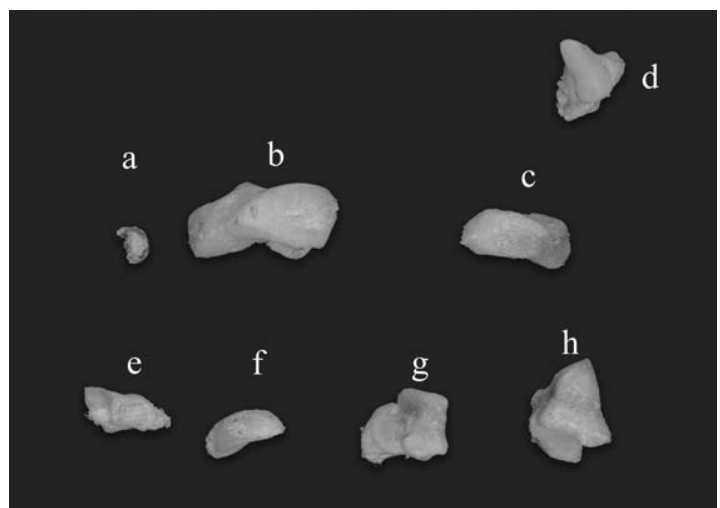

Figure 9. Individual carpal bones. a. Sesamoid bone. b. Radial carpal bone. c. Ulnar carpal bone. d. Accessory carpal bone. e. Carpal bone 1. f. Carpal bone 2. g. Carpal bone 3. h. Carpal bone 4 .

prominent palmar-lateral tubercle seen clearly radiographically (Fig. 10). The distal heads of Mc II-V bared trochlear-like articulation facets for articulation with the proximal phalanx and the paired, crescent-shaped proximal sesamoid bones of each digit (Fig. 12). Minimal differences were detected in the metacarpal region when compared to the domestic carnivores, except the reduction of Mc I and the complete absence of the first digit in the suricate. There was a variation in the length of the respective metacarpal bones. Mc III was the longest and $\mathrm{Mc} \mathrm{V}$ the shortest, with the joints in the corresponding digits being located most distally or most proximally, respectively (Fig. 10).

Metacarpal I: The supinated oblique view of the manus (Fig. 11) allowed the palmar-medial aspect of the carpo-metacarpal region to be highlighted. The oval-shaped Mc I showed some variation in shape, with a hook-like process of varying length seen distally. This process was markedly elongated (twice the length of the Mc I bone itself) in one mature animal (range $2-5 \mathrm{~mm}$ in length). It could be seen that Mc I articulated only with the medial aspect of the first carpal bone, and did not articulate with Mc II or the radial carpal bone. The large palmaro-medial process of the radial carpal bone was clearly seen extending medially beyond the level of the radial styloid process. The small sesamoid bone was consistently located adjacent to the radial carpal bone.

The digits: The elongated digits in the suricate lay close and parallel to each other and formed a slender manus region, which could be appreciated radiographically on the true DPa view (Fig. 10). 


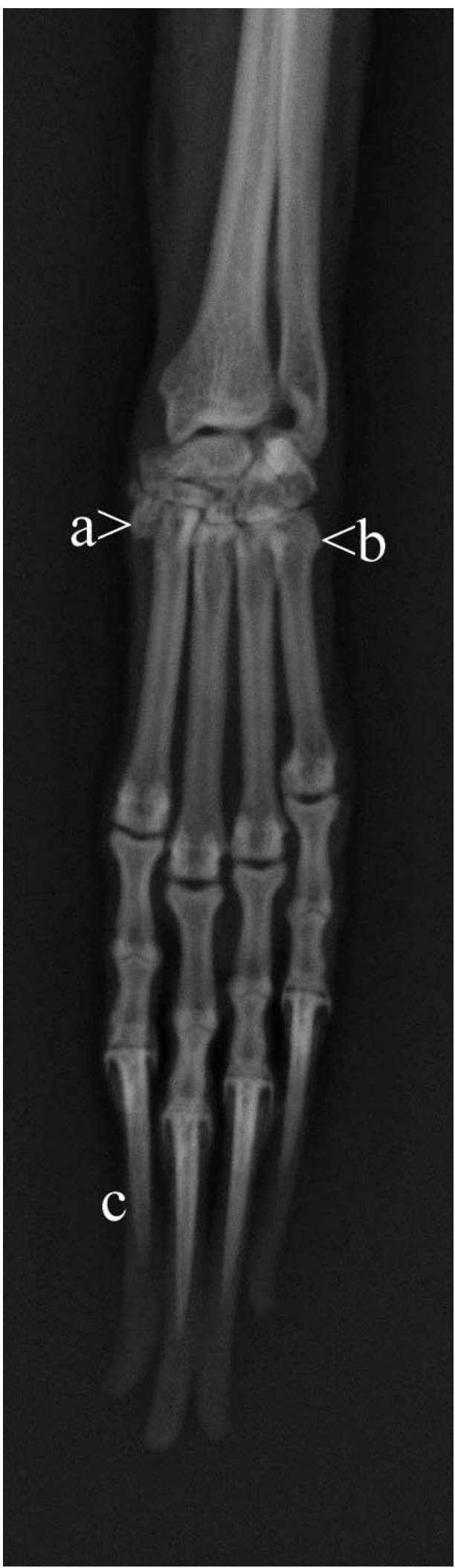

In comparison, the digits of the cat are more splayed. Phalanx proximalis and Phalanx media were both narrow rod-shaped bones in the suricate. The variation in length of the various proximal and middle phalanges was minimal. The base of Phalanx distalis (P3) was expanded palmarly to form the prominent, elongated oval Tub. flexorium for insertion of the deep digital flexor tendons. The Proc. unguicularis was very long and narrow with flattened axial and abaxial surfaces. The process was curved and convex dorsally, with a pointed tip (apex) distally. The Crista unguicularis was a well-defined thin shelf of bone and formed a deep Sulcus unguicularis, for attachment of the long nails (Fig. 10). The markedly elongated ungula process (and nails) was a characteristic that distinguished the suricate from the domestic cat and dog. In one dissected specimen, small cartilaginous Ossa sesamoideum distale were present in the flexor tendons of third, fourth, and fifth digits.

\section{Measurements and physes}

There were no significant differences $(P>0.05)$ between the measurements made on the bone specimens and the radiographs, and all measurements were combined into one group. Radiographic magnification was thus negligible because of the small size and positioning of the thoracic limb. The humerus, radius, and ulna (excluding the olecranon) were all of similar length in the suricate but were longer in relation to the scapula. Reference values for the long bones of the thoracic limb are given in Table 1.

The limited number of immature specimens in the radiographic study precluded any valid determination of physeal closure times for the suricate. In the two immature animals (9-11 mo); however, the distal ulnar and radial physes were still open and both had a transverse alignment similar to the domestic cat, but unlike the dog, where there is a conical-shaped physis in the ulna. The proximal humeral physis also showed a small remnant open physeal line in these animals. All the other physes and secondary ossification centers of the thoracic limb were closed.

Figure 10. True dorso-palmar view of the manus, showing the carpal articulations, the closely aligned and elongated digits with relative location of the digital joints. a. Os metacarpalis I. b. Tubercle of Os metacarpalis V. c. Elongated ungula process and claw. 


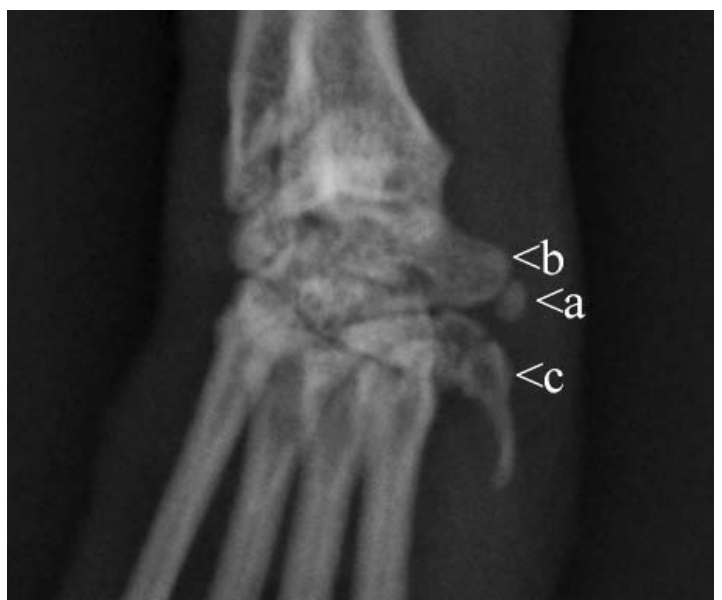

Figure 11. Supinated oblique view of the carpal region. a. Sesamoid bone. b. Palmaro-medial process of radial carpal bone. c. Os metacarpalis $\mathrm{I}$, articulating proximally with carpal bone 1 .

\section{Functional morphologic changes}

The unique osteology (and radiologic anatomy) as demonstrated in this study indicated certain morphologic changes associated with improved function of the shoulder, elbow, carpal, and digital flexor muscle groups. Osteologically, there was a marked prominence of the origin and insertion sites of all these flexor muscles when compared to the relative reduction and poor definition of the attachment sites of the corresponding extensor muscles.

Suricates' natural behavior and function demonstrated a predominance of shoulder flexion. The suricate commonly stands erect on the pelvic limbs to allow for improved visual observation of imminent danger. During this stance, the thoracic limbs are held alongside the trunk. In this study, the radiographic position of the limb with the shoulder in maximal flexion was equivalent to this natural position. The radiographic findings indicated great mobility of the shoulder joint and the scapula and ease of abduction from the thoracic wall. It is suggested that this allows prolonged flexion of the shoulder and the limb is easily held alongside the trunk.

Figure 12. Radiograph of an isolated digit. a. Os metacarpalis III. b. Proximal sesamoid bones. c. Phalanx proximalis. d. Phalanx media. e. Tub. flexorium of Phalanx distalis (P3). f. Elongated ungula process of P3.

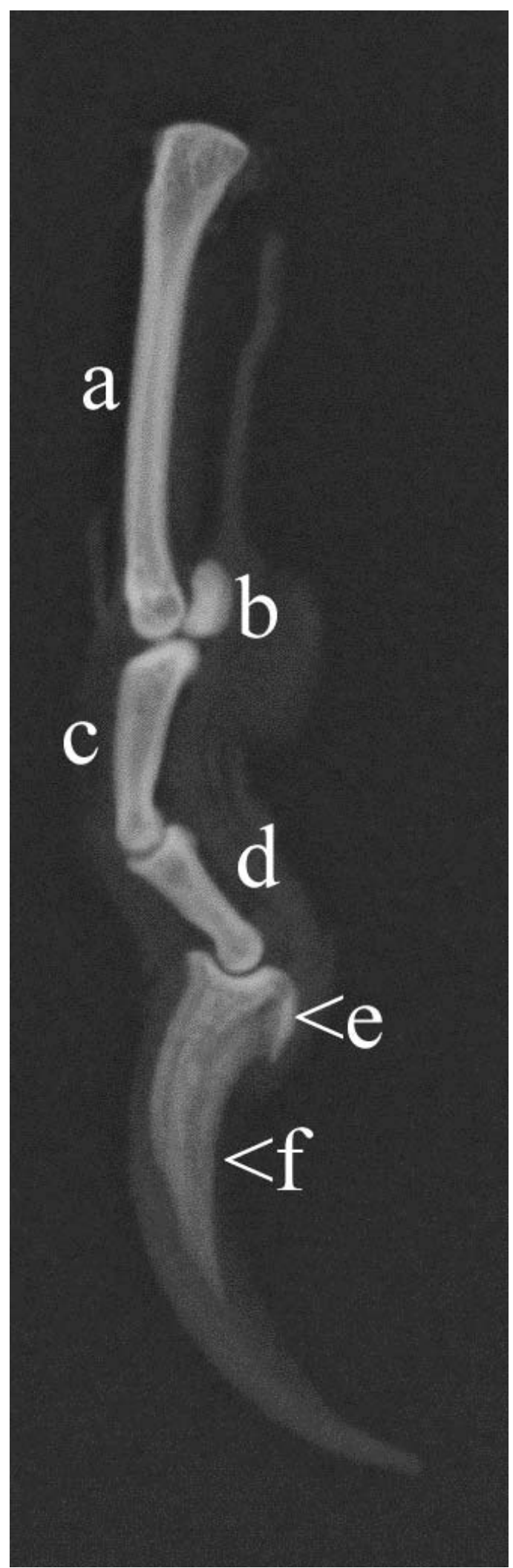


Table 1. Measurements of long bones of the thoracic limb.

\begin{tabular}{|c|c|c|c|c|c|c|c|c|c|c|c|c|c|}
\hline & \multicolumn{3}{|c|}{ Scapula } & \multirow{2}{*}{\multicolumn{3}{|c|}{ Humerus }} & \multirow{2}{*}{\multicolumn{3}{|c|}{ Radius }} & \multicolumn{4}{|c|}{ Ulna } \\
\hline & \multirow[b]{2}{*}{ Width } & \multirow{2}{*}{$\begin{array}{l}\text { Length } \\
\text { caudal }\end{array}$} & \multirow{2}{*}{$\begin{array}{l}\text { Length } \\
\text { at spine }\end{array}$} & & & & & & & Length to & Length & & \\
\hline & & & & Length & Breadth & Width & Length & Breadth & Width & olecranon & to $\mathrm{LCP}^{\mathrm{a}}$ & Breadth & Width \\
\hline Mean (mm) & 19 & 39 & 37 & 53 & 5 & 4 & 51 & 3 & 3 & 62 & 53 & 3 & 2 \\
\hline Range (mm) & $18-22$ & $36-42$ & $32-40$ & $50-57$ & $4-6$ & $3-5$ & $47-54$ & $2-3$ & $2-4$ & $57-66$ & $49-56$ & $3-4$ & $2-3$ \\
\hline SD & 1 & 2 & 2 & 2 & 0 & 0 & 2 & 0 & 0 & 2 & 2 & 0 & 0 \\
\hline
\end{tabular}

${ }^{a}$ LCP indicates lateral coronoid process.

Suricates have a superior digging ability and burrow complex tunnel systems underground for habitation. They also dig out prey, detected by smell, from the ground using the thoracic limbs. During the digging function, shoulder flexion was prolonged and maintained. Both thoracic limbs were usually used simultaneously, and the arc of movement was in the sagittal plane. The cranial extension of the limbs at the start of a digging cycle was limited. With the elbow joint maximally flexed, there was some extension of the shoulder joint to allow cranial movement of the limb until the digits were at the level of the muzzle. The powerful, caudal thrust of the limb then began. Contact between the long nails and the ground was maintained throughout, and achieved by prolonged flexion of the carpus and digital joints. Maintenance of elbow flexion and increasing flexion of the shoulder joint allowed for the strong caudal movement and thrust of the limb, required for the digging action, until the humerus lay horizontally alongside the trunk. The digits and nails were located between the pelvic limbs at the end of the caudal thrust. The increased mobility of the scapula and ease of abduction from the thoracic wall, as demonstrated in the radiographic study, most likely facilitated the caudal movement of the limb required to provide the powerful caudal thrust. Flexion of all the thoracic limb joints was thus maintained during the caudal movement. Prolonged, static, and strong contraction of all the flexor muscles of the thoracic limb was thus required for the performance of the digging action.

The absence of the clavicle in the suricate as a digging animal was unexpected. Mammals, such as the mole,${ }^{4}$ that use the forelimbs for burrowing usually have a well-developed clavicle, to provide extra stability to the shoulder joint. Without a clavicle, the shoulder region of the suricate should be less stable. However, as the suricate's thoracic limb moved only in a $\mathrm{CrCd}$ direction during digging, the shoulder stability can be maintained by muscle tone alone. The shoulder was held in a flexed position and thus supported and stabilized by the contraction of the strong shoulder flexor muscles. The lack of a clavicle likely also allows for a greater range of motion and ensures that the suricate is slender at the shoulder region to improve the ability to move through narrow burrows.

The shoulder flexor muscle function thus predominated over the shoulder extensors, and this enhanced function was reflected in various morphologic changes at the origin and insertion sites. The caudal angle of the scapula was prominent and the caudal border was thickened (origin the M. teres major). In addition, morphologic changes included the markedly deep Fossa infraspinata and the deep Facies m. infraspinati (M. infraspinatus), the relatively prominent Tuberositas teres minor, the prominence of the acromion with hamate process, the presence of the large suprahamate process (origin of the acromial part of the deltoid muscle), and the prominent deltoid tuberosity. It can be postulated that the function of the M. coracobrachialis, a shoulder extensor, is greatly reduced or even redundant in the suricate as reflected in the poorly defined origin site, namely the small coracoid process.

Elbow flexion contributed to the strength of the caudal thrust during digging. The prominence of the supraglenoid tubercle (origin site of the M. biceps brachii) was evident on the scapula in both the osteologic and radiologic studies. In addition, the insertion site at the Tuberositas radii was prominent and well defined. The brachial muscle also had a clearly defined insertion site on the proximal ulna. The relatively reduced function of the main elbow extensor, the triceps muscle, was reflected in the finding of the small infraglenoid tubercle of the scapula as described and the relatively smaller Tuber olecrani in the suricate when compared to the dog and cat.

The small lateral epicondyle of the humerus, the origin site for the carpal and digital extensors, reflected the relative reduction in function of these muscles in the suricate. The medial humeral 
epicondyle, the site of origin of all the carpal and digital flexors, was, however, markedly enlarged. Furthermore, the medial surface of the olecranon was deeply concave, which represented the origin site of the ulna head of the M. flexor carpi ulnaris. Specific modifications for the enhanced function of the deep digital flexor muscle were identified. The cartilaginous distal sesamoid bones provided a gliding surface for the tendons, and the welldeveloped Tub. flexorium provided for a strong insertion site on P3. Only one specimen was dissected in this study and further studies are required to confirm the presence of the distal sesamoid bones in all mature suricates.

The slender manus region with the reduction of the first metacarpal bone and absence of the first digit also contributes to an efficient digging action. In addition, the elongated ungula process of P3 in the suricate allowed for support of the longer nails designed for the digging function.

The radiologic study confirmed that the suricate has maintained the ability to supinate the radius. Morphologic changes identified, associated with this relative supination, included the articulation of the radial head with the capitulum of the distal humeral articulating surface, the caudo-lateral placement of the Margo interosseus on the radius, and the caudal location of the Tuberositas radii, which further promoted supination of the radius with contraction of the brachial biceps muscle. Suricates are also able to grasp and manipulate prey and other items with the thoracic limbs. The use of the strong carpal and digital flexors, along with the maintenance of the relative supination of the antebrachium and manus, allows suricates to perform these functions.

\section{CONCLUSIONS}

The bones of the thoracic limb of the suricate, when compared to the domestic carnivores, generally showed a closer resemblance to those of the cat, and shared a few characteristics of the bones of the dog. The unique osteology and radiologic anatomy of the suricate's thoracic limb indicated specific morphologic changes for improved function, namely superior digging ability and the supination of the antebrachium and manus. Findings can be applied in clinical zoologic medicine; further research of the suricate (and other related mammalian species) with regard to locomotion, function, behavior, and evolutionary adaptation of the skeletal system; and future paleozoologic studies.

Acknowledgments: The author acknowledges the National Museum, Bloemfontein, and the Transvaal Museum, Pretoria, for the loan of bone specimens; Dr. Adrian Tordiffe, National Zoological Gardens, Pretoria, for the provision of the live animals for radiography; Dr. Leon Venter, Department of Paraclinical Sciences, Faculty of Veterinary Science, University of Pretoria, for anesthesia and photography of the animals and other valuable guidance; and Charmaine Vermeulen, Department of Education Innovation, University of Pretoria, for bone photography.

\section{LITERATURE CITED}

1. Cespedes Diaz CM. A supratrochlear and a supracondylar foramen in the humerus of the mongoose (Herpestes auropunctatus). Folia Vet. 1975;19: 295-300.

2. Discovery Communications, Meerkat Manor, Animal Planet. [DVD] Silver Springs (MD); Discovery Communications; 2006.

3. International Committee on Veterinary Gross Anatomical Nomenclature. Nomina Anatomica Veterinaria [Internet]. 5th ed. Editorial Committee, World Association of Veterinary Anatomists; 2005 [cited 2007 August 30]. Available from: http://www.wava-amav.org

4. Skinner JD, Chimimba CT (eds.). The mammals of the southern African subregion. 3rd ed., Cambridge, United Kingdom: Cambridge Univ. Press; 2005. 2,428 p.

5. Stoskopf MK. Clinical imaging in zoological medicine: a review. J Zoo Wildl Med. 1989;20:396-412.

6. Van Staaden MJ. Suricata suricatta. Mamm Species. 1994;483:1-8.

Received for publication 29 November 2012 